\title{
CLINICAL PROFILE OF DIABETES MELLITUS IN CHILDREN AND ADOLESCENTS UNDER EIGHTEEN YEARS OF AGE
}

\author{
Fauzia Mohsin ${ }^{1}$, Bedowra Zabeen ${ }^{1}$, Rahelee Zinnat ${ }^{2}$ \\ Kishwar Azad ${ }^{1}$, Nazmun Nahar ${ }^{1}$ \\ ${ }^{1}$ Department of Paediatrics, Ibrahim Medical College \& BIRDEM \\ ${ }^{2}$ Department of Biochemistry and Cell Biology, Bangladesh Institute of Research and \\ Rehabilitation on Diabetes, Endocrine and Metabolic Disorders (BIRDEM)
}

\begin{abstract}
A total number of 125 patients with diabetes mellitus (DM) under eighteen years of age were admitted in the Paediartic department of BIRDEM hospital between January 2001 to October 2002. Eighty-eight patients (71\%) were newly detected. Female to male ratio was $3: 1$. Out of the total admission 38 (30.4\%) patients had type 1 DM (group 1), 37 (29.6\%) patients had fibrocalculous pancreatic FCPD diabetes (group II), 48 (38.4\%) patient had malnutrition modulated diabetes mellitus MMDM (group III) and 2 (1.6\%) patients had type 2. Mean age of onset was $9 \pm 3.9$ yrs in group I and $13 \pm 2.3$ yrs in group II and group III. All groups had very high glucose and HbA1c value at presentation. The mean fasting glucose (mmol/l) was $19 \pm 7.14,22.39 \pm 9$ and $19.54 \pm 7.9$ in group I, group II and group III respectively. The Mean HbA1c (\%) value in the three groups was $14.4 \pm 2.7,16.72 \pm 2.26$ and $15.27 \pm 3.05$ respectively. FCPD patients had poorest glycaemic status. Acute complications were more common in type 1 patients. Twelve (31.5\%) patients had diabetic ketoatin DKA and two (5\%) patients had hypoglycaemia in group I. Chronic complications were present in all three groups. MMDM patients had highest rate of complications. which was present in $2.6 \%, 21.6 \%$ and $33.3 \%$ patients in group I, group II and group III respectively. The rate of microalbuminuria was $5.3 \%$. $10.8 \%$ and $18.8 \%$ in the three groups respectively. The rate of neuropathy was $2.6 \%, 16.2 \%$ and $20.8 \%$ in the three groups respectively. Among the associated problems skin infection, pulmonary tuberculosis and bilateral parotid swelling were common. Malnutrition was present in 66\%, 86\% and $100 \%$ in group I, group II and group III respectively. Majority (50\% in group I, $91.6 \%$ in group II and $100 \%$ in group III) of our patients came from poor socio-economic background.
\end{abstract}

Ibrahim Med. Coll. J. 2007; 1(1): 11-15

\section{Introduction}

The increasing incidence of childhood and adolescent diabetes mellitus (DM) is a global phenomenon ${ }^{1}$. Type1 DM accounts for majority of childhood and adolescent onset diabetes seen in population of developed world. Type $2 \mathrm{DM}$ is also being increasingly reported in adolescents ${ }^{2}$. Malnutrition related diabetes mellitus (MRDM) was recognized by the 1985 WHO study group on Diabetes Mellitus as a major clinical type of diabetes distinct from type 1 and type 2 diabetes, unique to some tropical and developing countries ${ }^{3}$.
MRDM was subdivided into fibrocalculous pancreatic diabetes (FCPD) and protein deficient pancreatic diabetes (PDPD). Later the entity MRDM was deleted because the evidence that diabetes can be directly caused by protein deficiency was not convincing. FCPD has been reclassified as a disease of the exocrine pancreas ${ }^{4}$. The vast number of patients previously classified as PDPD has been subsequently renamed as malnutrition modulated diabetes mellitus (MMDM) after a prolonged debate in an international forum ${ }^{5}$.

Address for Correspondence:

Dr. Fauzia Mohsin, Department of Paediatrics, Ibrahim Medical College \& BIRDEM

122 Kazi Nazrul Islam Avenue, Shahbag, Dhaka-1000 
Bangladesh Institute of Research and Rehabilitation on Diabetes, Endocrine and Metabolic disorders (BIRDEM) is an internationally reputed tertiary level hospital for care of diabetic patients. By the end of 2003 , the number of registered diabetic patients in BIRDEM was 320,000 . The proportion of diabetics under eighteen years of age was found to be $1.58 \%$ among the registered cases of BIRDEM. It is expected that the under eighteen registered diabetic patients in BIRDEM represent the population of childhood and adolescent diabetics of Bangladesh. The present study was undertaken to examine the clinical profile of DM in children and adolescents in the Department of Paediatrics, BIRDEM.

\section{Patients and Methods}

Children and adolescents under eighteen years of age admitted consecutively in the Department of Paediatrics, BIRDEM from January 2001 to October 2002 were included in the study. Data were collected both retrospectively and prospectively. DM was diagnosed according to WHO criteria ${ }^{6}$. A uniform history sheet for each patient was completed including physical examination. Routine and relevant investigations were performed. All the patients were treated with subcutaneous insulin injection. Patients were classified as follows:

1. Type 1 DM (group I): Patients with early onset and/or with typical sign/symptoms of diabetes for a short duration were classified as type 1 diabetics. Patients presenting with diabetic ketoacidosis (DKA) were also included in this group.

2. FCPD: (group II): Patients having pancreatic calcification on x-ray or ultrasonography of abdomen in the absence of history of alcohol intake, hypercalcemia and biliary duct stone.

3. MMDM (group III): Patients with lean body mass (BMI less than $5^{\text {th }}$ centile for age), other evidence of malnutrition and no pancreatic calcification. They had signs/symptoms of diabetes for prolonged period but absence of ketosis.

4. Type 2 DM: Obese (BMI more than $95^{\text {th }}$ centile) and adolescent DM patients.

Microalbuminuria was defined as albumin/creatinine ratio (ACR): $30-300 \mathrm{mg} / \mathrm{gm}$ in spot urine.
Retinopathy was assessed by both direct and indirect ophthalmoscopy. Retinopathy was defined by presence of micro angiopathy in retinal vessels. Cataract was detected by slit lamp examination. Neuropathy was defined by absence of ankle jerk bilaterally or gross sensory disturbance in both feet.

All the data were expressed as mean \pm standard deviation, median (range) and/or number (\%) as appropriate. Statistical analysis was done by using SPSS for windows package. Appropriate statistical test of significance like unpaired t test was used as necessary. $\mathrm{P}<0.05$ was taken as minimal level of significance. Correlation study was done by Pearson correlation.

\section{Results}

A total of 125 patients were admitted during the study period. Eighty-eight $(71 \%)$ patients were newly detected. Female to male ratio was 3:1 among the newly detected patients. Out of the total admission 38 (30.4\%) patients had type 1 DM (group 1), 37 (29.6\%) patients had FCPD (group II), 48 (38.4) had MMDM (group III) and $2(1.6 \%)$ patients had type 2 DM. Among the newly detected cases the distribution was almost similar with $33 \%, 28.4 \%, 36.4 \%$ and $2.3 \%$ being type1 DM, FCPD, MMDM and type2 DM respectively.

All patients in group II and group III had typical symptoms of diabetes at diagnosis. In addition, 32\% of FCPD patients had history of recurrent abdominal pain. One patient in group I had nocturnal enuresis as the only presenting symptom. Seven (24\%) of type 1 diabetic patients developed DKA at first presentation, six of them had typical symptoms of DM for a short period (few days to few weeks) before developing DKA, but one patient presented with DKA only without preceding symptoms of diabetes. A substantial number of patients presented themselves with chronic complications such as cataract, microalbuminuria and neuropathy at diagnosis. Out of 25 cases of cataract, $16(64 \%)$ cases were newly detected. Ten $(66 \%)$ out of fifteen patients with microalbuminuria and $12(70 \%)$ out of seventeen patients with neuropathy were newly detected.

Mean $( \pm$ SD) age (yrs) of onset was $9 \pm 3.9,13 \pm 2.3$ and $13 \pm 2.3$ in group I, group II and group III respectively. Type 1 patients were significantly younger than the other two groups $(\mathrm{p}<0001$ in group I vs. group II and group III ). The youngest patient was 2 years old with type 1 diabetes. 
Table 1. Glycaemic status among the three groups

\begin{tabular}{lccc}
\hline Glyacemic control & Group I & Group II & Group III \\
\hline Fasting blood glucose (mmol/l) & $19 \pm 7.14$ & $22.39 \pm 9$ & $19.54 \pm 7.9$ \\
Blood glucose 2 hour after & $24.21 \pm 7.6$ & $31.07 \pm 7.1$ & $27.22 \pm 10.5$ \\
breakfast( mmol/l) & & & \\
HbA1c $(\%)$ & $14.49 \pm 2.7$ & $16.72 \pm 2.26$ & $15.27 \pm 3.05$ \\
\hline
\end{tabular}

Among the newly detected patients mean $( \pm S D)$ duration (months) of typical symptoms of DM prior to diagnosis was $2.0 \pm 2.9,6.3 \pm 9.6$ and $13 \pm 12$ in group I, group II and group III respectively. There was significant difference in duration of symptoms among the three groups $(\mathrm{p}=0.02$ in group I vs. group II; $p<0.0001$ in group I vs. group III; $p=0.03$ in group II vs. group III). MMDM group had longest duration of typical symptoms of diabetes.

Mean ( \pm SD) fasting blood glucose, glucose value two hours after breakfast and HbAlc values in the three groups are shown in Table 1.

The mean glucose value two hours after breakfast was significantly higher in FCPD patients compared to type 1 diabetic patients $(p=0.0003)$. HbAlc value was also significantly higher in FCPD patients compared to Type 1 diabetic patients $(\mathrm{p}<0.0001)$. There was no significant difference in glycaemic status between MMDM and Type 1 diabetic patients. When comparing between FCPD and MMDM patients, $\mathrm{HbA}$ 1c value was significantly higher in FCPD patients $(\mathrm{p}=0.033)$.

Acute complications were more common in type 1 patients. In group I, twelve (31.5\%) patients had DKA and $2(5 \%)$ patients had hypoglycaemia. The first presentation was with DKA in seven (24\%) type 1 diabetic patients. Only one (2.7\%) FCPD patient had DKA along with septicaemia.

Chronic complications (Fig-1) were found in all the three groups. Various complications among the three groups are shown in Fig 1.

Chronic Compliocations ( in percentage) among the three groups

Fig. 1

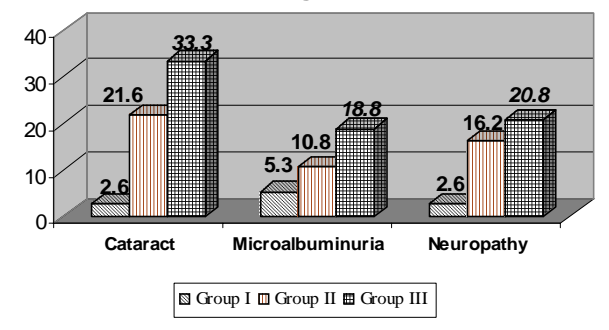

Table 2. Distribution of associated problems as among the three groups

\begin{tabular}{lccc}
\hline Problems & Group & Group II & Group III \\
\hline Pulmonary Tuberculosis & $1(2.6 \%)$ & $1(2.7 \%)$ & $7(14.6)$ \\
Skin infection & $19(2.6 \%)$ & $3(8.1)$ & $5(10.4 \%)$ \\
Bilateral parotid swelling & 0 & $1(2.7)$ & $12(25 \%)$ \\
\hline
\end{tabular}

Two (2.7\%) FCPD patients had early retinopathy of one eye, one (2\%) MRDM patient had bilateral progressive retinopathy (not shown in Fig. 1).

No significant correlation was found between any of the complication and duration of diabetes, duration of symptom, glycaemic status, age of onset or age on admission. There were some associated problems as shown in table 2.

Family history of diabetes was present in eight (22.2\%) type 1 , one (2.6\%) FCPD and three (8.1\%) MMDM patients.

Malnutrition was present in $66 \%, 86 \%$ and $100 \%$ of patients in group I, group II and group III respectively. Both of the type 2 patients were obese with BMI more than $95^{\text {th }}$ centile for age.

Majority (50\% in group I, $91.6 \%$ in group II and $100 \%$ in group III) of our patients came from poor socioeconomic background.

Only two of our patients had type 2 diabetes: a 16 year old female and another 13 year old male, both were from good socioeconomic background.

During the study period 2 patients died. One patient with type 1 DM died of DKA. One patient with FCPD died of septicaemia and pneumonia. She developed DKA before death.

\section{Discussion}

In this large cohort of young diabetics, MMDM was commonest succeeded by FCPD and Type1 diabetes among the newly diagnosed patients, only two patients had type 2 diabetes. This picture is quite different from western and developed countries. Cases of FCPD have been described in several other countries including India, Brazil, Indonesia, Jamaica, Madagascar, Nigeria, Sri Lanka, Uganda, Zair and Zambia ${ }^{3}$. MMDM has also been described in a number of Asian, African, Latin American and Caribbean countries ${ }^{7}$. 
There was a female preponderance, consistent with our previous studies in BIRDEM ${ }^{8}$. Patients in FCPD and MMDM groups were mostly adolescents (mean age $13 \pm 2.3$ years). In the majority of FCPD patients diagnosis is made between the ages of 20 and 40 years of age but onset in childhood, in infancy and at older age groups are not uncommon ${ }^{9-11}$. Onset of MMDM is commonly between 10 to 30 years but onset at preschool age and over the age of 30 years can also occur $^{7}$. There is said to be a bimodal distribution for type 1 diabetics, with a minor peak at 4 to 6 years and a major peak at 10 to 14 years. In our study population, the mean age onset of type 1 diabetics was $9 \pm 3.9$ years.

There was a significant difference in duration of symptoms of diabetes between type 1 and the other two groups. Specially MMDM group had quite a long duration of typical symptoms of diabetes, even up to three years but only few of them (21\% compared to $72 \%$ in type 1 patients) had acetonuria despite very high blood glucose level at presentation. None of them presented with DKA. Although FCPD is a definite separate entity, the classification of MMDM has still remained an enigma. It is possible that MMDM is actually a slow onset type 1 diabetes, whose clinical pictures have been modified by prolonged under nutrition to give rise to unique features such as insulin resistance and ketosis resistance. Continued presence of residual Beta cell functions, deficiency in hepatic carnitine and failure to the rise of glucagon have also been implicated for suppressed tendency to generate ketosis $^{7,12,13}$.

The very high blood glucose and $\mathrm{HbA} 1 \mathrm{c}$ in the three groups is not unexpected, as most of these are values at first presentation of diabetes. Glycaemic control was worst in FCPD patients.

Among the various complications, cataract was the commonest. Although rare in the developed world, cataract has been described very soon after the onset of diabetes, specially if there has been a slow or prolonged onset of diabetes ${ }^{14}$. Premature cataract has been reported in 10 to $15 \%$ of MMDM patient in India ${ }^{7}$. Microalbuminuria was found in 5.3 to $18 \%$ of our study population in the three groups. Microalbuminuria has been reported in 4 to $20 \%$ of children mostly after the age of $12-15$ years in various centers ${ }^{15,16}$. Neuropathy ranged from 2.6 to $20.8 \%$ in our 3 groups. Reported prevalence of neuropathy is from 16 to $51 \%$ among childhood and adolescent diabetics in various centers ${ }^{17,18}$. Although neuropathy is mostly subclinical in adolescents in western world, most of our patients had symptoms like severe pain and burning sensations of extremities.

A striking feature of our study population was the presence of microvascular complications (microalbuminuria and neuropathy) even at presentation and a very high rate of complication in the MMDM group. Possible explanation is that they may have very slow onset diabetes or have remained undiagnosed for a long time. This is possible because despite having severe hyperglycaemia they are ketosis resistant ${ }^{19}$.

Majority of our patients suffered from malnutrition. While malnutrition is inevitable in MMDM patients, the same may not be true in FCPD patients. Recent reports from different parts of India suggest a change in clinical features of FCPD patients because of improved nutritional status. Overt malnutrition was observed in $25 \%$ of FCPD patients in India ${ }^{20}$.

Positive family history was present in $22.2 \%, 2.7 \%$ and $8.1 \%$ Type 1, FCPD and MMDM patients respectively. Although genetic factor is important for the development of type 1 diabetes, positive family history is uncommon $(<10 \%)$ in MMDM suggesting that the disease is more likely environmental than genetic $^{7}$. Familial aggregation of FCPD patients have been reported from India and Bangladesh suggesting genetic susceptibility ${ }^{21,22}$.

\section{Conclusion}

The overall clinical presentation of our diabetic population is somewhat different from that of developed and western world. Acute complications such as DKA and hypoglycaemia are more common in Type 1 patients as found worldwide but the high rate of complication even at presentation among FCPD and MMDM patients and their poor nutritional status are of concern.

Measurement of autoantibody and C-peptide is necessary for proper classification of our diabetic patients, rather than depending on clinical presentation alone, although this might not be always possible in our present context. Finally the need for a population based, longitudinal study is stressed to evaluate the proper incidence and pattern of childhood and adolescent diabetes and its complications. Early 
detection and treatment of diabetes and its complications prevents or delays the deleterious consequences of diabetes and ensures that patients lead a normal life.

\section{References}

1. Ruwaard D, Hirasing RA, Reeser HM et al. Increasing incidence of type 1 diabetes in the Netherlands. The second nationwide study among children under 20 years of age. Diabetes care 1994; 17: 599-601.

2. Pinhas-Hamiel O, Dolan LM, Daniels SR et al. Increased incidence of NIDDM among adolescents. J Pediatr 1996; 128: 608-615.

3. WHO technical report series 727. Diabetes Mellitus. World Health Organization, Geneva, 1985.

4. Report of the Expert Committee on the diagnosis and classification of diabetes mellitus. Diabetes Care 1994; 21: 5-19.

5. Tripathy BB, Samal KC. Overview consensus statement on Diabetes in Tropical areas. Diabetes / Metab Rev 1997; 13: 63-7.

6. Report of the Expert Committee on the Diagnosis and Classification of Diabetes Mellitus. Diabetes Care 1999; 20 (Supp11): S5.

7. Samal KC, Kanungo A, Sanjeevi CB. Clinicoepidemiological and Biochemichal profile of Malnutrition-Modulated Diabetes Mellitus. Ann. N.Y. SCI. 2002; 958: 131-137.

8. Haque A, Azad K, Abdullah AHM. Diabetes in children and adolescents in Bangladesh. Diabetes Endocr J 2000; 16: 728 (ref type-abstract).

9. Mohan V, Ramachandran A, Viswanathan N. Childhood onset fibrocalculous pancreatic diabetes. Int J Diabetes Dev Countries 1990; 10: 24-26.

10. Premalatha G, Mohan V. fibrocalculous Pancreatic Diabetes in Infancy: two case reports. Diabetes Res Clin Pract 1994; 25: 137-40.
11. Mohan V, Suresh S, Indrani S et al. Fibrocalculous Pancreatic Diabetes in the elderly. J Assoc Phys Ind 1989; 37: 342-344.

12. Sood R, Ahooja MMS, Karnarkar MG. Serum Cpeptide levels in young ketosis resistance diabetics. Ind J Med Res 1983; 78: 661-664.

13. Mohan V, SnehalataC, Ramachandran A et al. Plasma glucagons response in tropical fibrocalculous pancreatic diabetes. Diabetes res Clin Pract 1990; 9: 97-101.

14. Datta V, Swift PG,Woodruff GH et al. Metabolic cataracts in newly diagnosed diabetes. Arch Dis Child 1997; 76: 118-120.

15. Donaghue KC, Fairchild JM, Chan A et al. Diabetes complication screening in 937 children and adolescents. JPEM 1999; 12: 185-92.

16. Cook JJ, Daneman D. Microalbuminuria in adolescents with insulin-dependent diabetes mellitus. Am J Dis Child 1990; 144: 234-237.

17. Ludvigsson J, Johanesson G, Heding L et al. Sensory nerve conduction velocity and vibratory sensibility in juvenile diabetics. Relationship to endogenous insulin. Acta Paediatr Scand 1979; 68: 739-48.

18. Young RJ, ewing DJ, Clarke BF. Nerve function and metabolic control in teenage diabetics. Diabetes 1983; 32: 142-7.

19. Hugh-Jones P. Diabetes in Jamaica. Lancet 1955; 2 : 891.

20. Mohan V, Mohan R, Susheela L et al. Tropical pancreatic diabetes in South India: heterogeneity in clinical and biochemichal profile. Diabetologia 1985; 28: 229-232.

21. Chowdhury ZMd, McDermott MF, Davey S et al. Genetic susceptibility to fibrocalculous pancreatic diabetes in Bangladeshi subjects: a family study. Genes and Immunity 2002; 3: 5-8.

22. Mohan V, Chari ST, Hitman S et al. Familial aggregation in Tropical Fibrocalculous Pancreatic Diabetes, Pancreas 1989; 4: 690-693. 